\title{
Patient and Caregiver Treatment Preferences in Type 2 and Non-ambulatory Type 3 Spinal Muscular Atrophy: A Discrete Choice Experiment Survey in Five European Countries
}

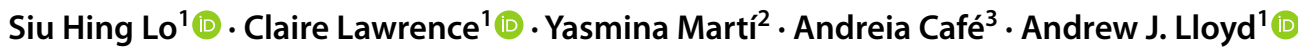

Accepted: 21 November 2021 / Published online: 13 December 2021

(c) The Author(s) 2021

\begin{abstract}
Background Spinal muscular atrophy (SMA) is a rare neuromuscular disease that affects motor neurons, resulting in progressive skeletal muscle weakness and atrophy.

Objective The aim of this study was to examine treatment preferences of patients and caregivers of patients with Type 2 and non-ambulatory Type 3 SMA in the Netherlands, Belgium, Finland, Ireland and Portugal.

Methods A discrete choice experiment (DCE) survey was developed to elicit the preferences of adult patients and caregivers regarding different treatment aspects of SMA. This survey built on the design of a similar study undertaken in the UK. The DCE described choice questions in terms of attributes and levels combined using a D-efficient design. The attributes described improvements or worsening in motor and breathing function. The mode of treatment administration (intrathecal injection, single intravenous infusion or regular oral therapy) was described. Treatment risks and side effects related to currently available treatments including risk of liver injury, fatigue, headache, nausea, diarrhoea and rash were described. Lastly, an attribute described whether a treatment had evidence of treatment effectiveness in different SMA types. Participants were recruited via patient advocacy associations to complete an online survey. A clustered conditional logit model was used to estimate treatment preferences.

Results Participants $(n=65)$ were 4.8 times and 8.1 times more likely to choose a treatment with stable or improved (vs worse) motor function, respectively. Similarly, participants were 4.3 times and 5.8 times more likely to choose stable or improved (vs worse) breathing function, respectively. Treatments with a risk of liver injury, fatigue, headache and nausea were 1.6 times less likely to be chosen than treatments with a risk of diarrhoea and rash. Treatments with demonstrated effectiveness in Type 1 SMA only were 2.3 times less likely to be chosen than those with demonstrated effectiveness in Types 1-3 SMA. Treatments administered via intrathecal injections were also 1.8 times less likely to be chosen than daily oral treatments.

Discussion Study results show the importance of improvement as well as stabilisation of motor and breathing function to patients and caregivers, and a preference for oral treatments, treatments with demonstrated effectiveness in Types 2-3 SMA, and avoidance of liver injury risk.
\end{abstract}

\section{Introduction}

Spinal muscular atrophy (SMA) is an autosomal recessive neuromuscular disease characterised by degeneration of alpha motor neurons in the spinal cord, resulting in

Siu Hing Lo

siuhing.lo@acasterlloyd.com

1 Acaster Lloyd Consulting Ltd, London, UK

2 F. Hoffmann-La Roche Ltd, Basel, Switzerland

3 F. Hoffmann-La Roche Ltd, Amadora, Portugal progressive proximal muscle weakness and paralysis [1]. Two genes are implicated in the development and severity of SMA; survival of motor neuron 1(SMN1) and SMN2. Loss of, or defect in SMN1 results in reduced production of SMN protein, which is critical for the maintenance of motor neurons. The severity of SMA also often decreases with increasing copy numbers of the SMN2 gene [2].

SMA is classified into phenotypes based on age at onset and highest motor milestone achieved. Type 1 SMA is the most common and severe, affecting approximately $50 \%$ of patients. Infants with Type 1 SMA typically have onset of clinical signs by 6 months of age, never develop the ability 


\section{Key Points for Decision Makers}

Adult patients and caregivers of patients with Type 2 and non-ambulatory Type 3 SMA strongly preferred treatments that stabilised or improved motor and breathing function.

Participants also preferred a daily oral treatment compared with other modes of administration.

Participants preferred treatments with demonstrated evidence of effectiveness in Types 2-3 SMA and preferred to avoid risk of liver injury.

to sit independently and generally do not survive beyond 2 years of age, if no intervention is provided [1]. Type 2 SMA onset occurs between 7 and 18 months of age. Patients typically develop the ability to sit independently and some are able to stand, with $70 \%$ survival at 25 years of age. Type 3 SMA onset occurs between 18 months and 17 years of age and life expectancy is into adulthood. These patients achieve the ability to walk, but this ability may be lost over time. Natural history data demonstrate that SMA is a progressive disease, with function declining over time [3-6]. For patients with Type 2 or Type 3 SMA specifically, the greatest unmet need is access to treatments that reduce musculoskeletal complications, improve or maintain respiratory and motor function, and support independence [7]. In addition, survey research and qualitative research with patients and caregivers suggest that stabilisation of the disease course would represent important progress [8-10].

Three treatments for SMA are approved in Europe. Nusinersen (SPINRAZA $®$ ), the first approved treatment indicated for the treatment of 5q SMA, has been shown to halt disease progression and can significantly improve motor function $[11,12]$. Nusinersen is administered via intrathecal injection (lumbar puncture) directly to the cerebrospinal fluid around the spinal cord, limiting its distribution to the central nervous system. The treatment schedule involves four loading doses (days 0, 14, 28 and 63) followed by long-term maintenance doses every 4 months. There is a risk of adverse reactions occurring with the lumbar puncture procedure (e.g. headache, back pain, vomiting) and patients who have scoliosis or spinal fusion might be precluded from receiving nusinersen.

In 2020, onasemnogene abeparvovec (ZOLGENSMA $®$ ) received conditional approval in the $\mathrm{EU}$ as a gene therapy for patients with Type 1 SMA or for people with bi-allelic mutation in the SMN1 gene and up to three copies of the $S M N 2$ gene [13]. Onasemnogene abeparvovec is delivered in a clinical setting using a single intravenous infusion. In a trial of 15 patients with Type 1 SMA, those treated with onasemnogene abeparvovec survived longer and displayed improvements in motor function outcomes compared with historical cohorts [14]. Evidence for the efficacy of onasemnogene abeparvovec has been primarily shown in Type 1 SMA. However, recent evidence suggests onasemnogene abeparvovec is also effective at improving motor function in presymptomatic patients with bi-allelic SMN1 deletions and two or three copies of the SMN2 gene $[15,16]$. Common adverse reactions include increased transaminases and troponin-I, and acute serious liver injury can occur; liver function monitoring and treatment with corticosteroids in conjunction with onasemnogene abeparvovec are therefore recommended [13].

Finally, risdiplam (EVRYSDI $\left.{ }^{\circledR}\right)$ is approved as a treatment for patients 2 months of age and older with a clinical diagnosis of Type 1-3 SMA or with one to four $S M N 2$ copies. Risdiplam is an SMN2 splicing modifier, which is given daily by mouth (or g-tube) and distributes throughout the body. Risdiplam has been studied in infants, children and adults with SMA who exhibit a broad range of disease severity and functional ability. Data from the pivotal FIREFISH trial of risdiplam in patients with Type 1 SMA demonstrate improvements in motor function, including the ability to sit without support at 24 months compared with the natural course of Type 1 SMA [17]. Data of patients with Type 2-3 SMA from the SUNFISH trial indicate that changes in motor function were significantly greater in the treatment group compared with the placebo group at the 12-month follow-up [18]. The most common adverse drug reactions related to risdiplam in the SUNFISH clinical trial were diarrhoea and rash [26].

Previous research has explored patient and caregiver preferences for different treatment options. In a recent UK study, discrete choice experiment (DCE) surveys were developed for adult patients and caregivers of patients with Type 2 and non-ambulatory Type 3 SMA based on treatment profiles for risdiplam and nusinersen [19]. Caregivers placed highest value on improving motor and respiratory function. Patients placed most value on stabilising/avoiding worsening motor and respiratory function. Another study in the US examined patient and caregiver preferences for key attributes of treatments using a DCE in Type 1-4 SMA [20]. Patients and caregivers placed high value on treatments that improve motor and breathing function, that are indicated across all ages, whose administration is oral or a one-time infusion, and that have minimal risk profiles. To our knowledge, research has not yet examined patient and caregiver preferences comparing treatment characteristics of risdiplam, nusinersen and onasemnogene abeparvovec within European populations.

The present study aims to assess the preferences of adult patients and caregivers/parents of patients with Type 2 and non-ambulatory Type 3 SMA for treatment characteristics in the Netherlands, Belgium, Portugal, Ireland and Finland. 


\section{Methods}

\subsection{Study Design}

The main survey comprised clinical and demographic measures of the patient and, if applicable, demographics of the caregiver (caregiver participants only), and a DCE. DCEs are a widely used method for eliciting patient treatment preference, where hypothetical treatments are described in terms of their treatment characteristics ('attributes' [21, 22]). Attributes were based on known SMA treatments at the time of study development. In the DCE, participants were first presented with descriptions of attributes and attribute levels, followed by 15 choice questions. The choice questions asked participants to choose between two hypothetical treatments, varying by 'treatment attributes' representing treatment benefits, side effects, mode of administration, and treatment evidence available for different SMA types. The relative importance of different attributes was estimated from the choices participants made [23].

\subsection{Participant Recruitment and Procedures}

Participants were recruited in the Netherlands, Belgium, Finland, Ireland, and Portugal. They were recruited through patient advocacy associations in each of the countries. Potential participants were contacted via email or advertisements on their social media channels. The following eligibility criteria were used:

- A patient self-reported or caregiver proxy-reported diagnosis of Type 2 (age of onset between 7 and 18 months) or Type 3 SMA (age of onset between 18 months and 17 years)

- The patient had to be 'non-ambulatory', defined as being unable to walk more than ten steps without a form of assistance

- Adult ( $\geq 18$ years) patients or caregivers/parents of a child with Type 2 or 3 SMA

Potential participants first completed a screening questionnaire, with questions used to determine eligibility for study participation. Eligible participants were provided with an information sheet about the study which described the study content, study procedures and their rights as participants, and were asked to complete a consent form if they agreed to take part. Consenting participants then proceeded to the main survey. Participants did not receive remuneration for their study participation.

To accommodate country-specific ethical and data protection requirements, minor adaptations to the invitation text, consent form and the introduction to the main survey were made. All study materials were translated and certified by a translation agency and members of local patient associations.

The study survey was administered online, with appropriate local language versions provided for each of the study countries. The participants were first asked to read the participant information sheet and then to indicate whether they consented to take part. Following consent, participants completed the study screener, and if eligible, were directed to the main study survey. The recruitment letter and study survey can be viewed in the electronic supplementary material.

\subsection{Institutional Review Board (IRB) Approval}

All study materials were submitted to the Western Institutional Review Board (WIRB) in the US for ethical review. The WIRB reviewed the documents and declared the study exempt from ethical review on 19 August 2020 (submission number: 2639333-44664387).

\subsection{Treatment Attributes and Attribute Levels}

The DCE survey aimed to capture treatment attributes (i.e. treatment characteristics) relevant to patients and caregivers of patients with Type 2 and non-ambulatory Type 3 SMA when choosing among different treatment options. The DCE survey design and choice of attributes were based on a previous SMA DCE study conducted in the UK. The original selection of attributes was guided by the literature, input from three clinical experts, and treatment characteristics for nusinersen and risdiplam [19]. Details on the natural history of the main types of SMA and key functional impairments related to patients' motor and respiratory function as well as regulatory documents for nusinersen and the risdiplam profile were based on the literature $[1,24,25]$. Three SMA clinical experts were interviewed to obtain feedback on the potential treatment attributes. Specifically, clinicians were asked to comment on the clinical relevance of potential attributes and overlap between attributes. For motor function, respiratory function and treatment-related reactions, specific feedback on the clinical accuracy of the range presented in the attribute levels was also elicited. Risk of infection and other pulmonary problems (other than breathing) were also discussed as potential attributes but the clinical experts considered them to be less relevant and so they were not included. No specific feedback was sought on the levels for other treatment attributes because these were based on known characteristics of nusinersen and likely characteristics of risdiplam.

The study design was updated to include treatment attributes reflecting key treatment characteristics for onasemnogene abeparvovec, as detailed in its Summary 
of Product Characteristics, and to reflect the most recent available data on nusinersen and risdiplam $[11,13,26]$. An overview of attributes and attribute levels that informed the current study design is provided in Table 1; the overview is also described hereafter alongside a summary of the updates that were made to reflect onasemnogene abeparvovec treatment characteristics and recent data on nusinersen and risdiplam.

a. Motor function was described 'better by one level', 'same as the current level' or 'worse by one level' in a year's time on the motor function scale (Fig. 1). This attribute was retained from the previous study, as stabilisation or improvement in motor function remained a key treatment benefit across all three treatment options.

b. Breathing function was described as 'better', 'the same' or 'worse' in a year's time. This attribute was retained from the previous study, as stabilisation or improvement in breathing function remained a key treatment benefit across all three treatment options.

c. Treatment administration was described as a(n) 'daily oral liquid', 'injection into the spine every 4 months' or 'single intravenous infusion and oral medication before and after the infusion' $[11,13,26]$. An additional attribute level reflecting onasemnogene abeparvovec administration ('single intravenous infusion and oral medication') was added to the two levels describing administration of nusinersen and risdiplam in the previous study.

d. Safety and side effects was described as 'risk of diarrhoea and rash' [26], 'fever, headache, vomiting and/or body pain for 1-2 days every 4 months' [11] and 'risk of liver injury, and fatigue, headache and nausea for 2 months' [13]. A single 'safety and side effects' attribute was created representing key safety and tolerability aspects for each of the three treatment options. For

Table 1 SMA DCE survey: overview of selected DCE attributes

\begin{tabular}{|c|c|c|c|}
\hline & Level 1 (Reference category) & Level 2 & Level 3 \\
\hline Motor function ${ }^{\mathrm{a}}$ & $\begin{array}{l}\text { Motor function will be worse by } \\
\text { one level in a year's time }\end{array}$ & $\begin{array}{l}\text { Motor function will be the same as } \\
\text { the current level of function in a } \\
\text { year's time }\end{array}$ & $\begin{array}{l}\text { Motor function will be better by one } \\
\text { level in a year's time }\end{array}$ \\
\hline Breathing function $^{a}$ & $\begin{array}{l}\text { Breathing function will be worse } \\
\text { in a year's time }\end{array}$ & $\begin{array}{l}\text { Breathing function will be the } \\
\text { same in a year's time }\end{array}$ & $\begin{array}{l}\text { Breathing function will be better in a } \\
\text { year's time }\end{array}$ \\
\hline Treatment administration & $\begin{array}{l}\text { Treatment is taken by an oral } \\
\text { liquid daily or by gastric tube at } \\
\text { home }\end{array}$ & $\begin{array}{l}\text { Injection into the spine (lower } \\
\text { back) in hospital every } 4 \text { months }\end{array}$ & $\begin{array}{l}\text { Once-only injection into a vein in } \\
\text { hospital with daily oral tablets for } \\
2 \text { months }\end{array}$ \\
\hline Treatment risks and side effects & $\begin{array}{l}\text { A } 17 \% \text { ( } 1 \text { in } 6 \text { ) risk of diarrhoea } \\
\text { and rash }\end{array}$ & $\begin{array}{l}\text { Most patients will have fever, head- } \\
\text { ache, vomiting and/or body pain } \\
\text { for 1-2 days every } 4 \text { months }\end{array}$ & $\begin{array}{l}\text { A } 10 \% \text { ( } 1 \text { in } 10) \text { risk of liver injury. } \\
\text { Fatigue, headache and nausea for } \\
2 \text { months }\end{array}$ \\
\hline Treatment evidence & $\begin{array}{l}\text { Effectiveness has been demon- } \\
\text { strated in patients with Types } 1 \text {, } \\
2 \text { and } 3 \text { SMA }\end{array}$ & $\begin{array}{l}\text { Effectiveness has been demon- } \\
\text { strated in patients with Types } 1 \\
\text { and } 2 \text { SMA }\end{array}$ & $\begin{array}{l}\text { Effectiveness has been demonstrated } \\
\text { in patients with Type 1 SMA }\end{array}$ \\
\hline
\end{tabular}

$D C E$ discrete choice experiment, SMA spinal muscular atrophy

${ }^{a}$ Motor and breathing functions were described as the 'average treatment effect/effectiveness on motor/breathing function' in the full text

Fig. 1 Motor function scale

\begin{tabular}{|l|l|}
\hline 1 & Cannot sit \\
2 & Can sit with some support (e.g. with back support or arm support) \\
3 & Can sit independently for a few seconds \\
4 & Can sit independently for a longer period of time but cannot stand \\
5 & Can sit independently and stand with assistance, but cannot walk \\
6 & Can sit independently and stand and walk with assistance \\
7 & Can sit, stand and walk independently for a few steps (less than 10 metres) \\
8 & In hypothetical DCE choice questions only \\
& Can sit, stand and walk independently over longer distances (more than 10 metres) \\
\hline
\end{tabular}

$\underline{\text { Underlined text used as short form in main text }}$ 
nusinersen, the previous study results had shown that 'treatment reactions' (i.e. reactions that patients commonly experience with an intrathecal injection) had a small effect on caregiver and patient preferences at most. The original 'treatment reactions' attribute was therefore converted to an attribute level of 'fever, headache, vomiting and/or body pain for 1-2 days every 4 months', representing the typical duration of reactions to intrathecal injections [11]. For risdiplam, 'risk of diarrhoea and rash' was added based on recent clinical evidence suggesting that the most common adverse effects are diarrhoea and rash [26]. For onasemnogene abeparvovec, 'risk of liver injury, and fatigue, headache and nausea for 2 months' was added as a new attribute level [13].

e. Treatment evidence was described as 'demonstrated effectiveness in Type 1 SMA', 'demonstrated effectiveness in Types 1 and 2 SMA', and 'demonstrated effectiveness in Types 1, 2 and 3 SMA' $[11,13,26]$. This attribute was newly added because the onasemnogene abeparvovec approval included all patients with biallelic mutation in the SMN1 gene and up to three copies of the SMN2 gene, but trial evidence only included patients with Type 1 SMA [14-16]. In contrast, there is trial evidence to support the use of nusinersen and risdiplam in Types 1-3 SMA [12, 17, 18]. This is important for different reasons. First, patients, caregivers and other decision makers have to make a decision regarding whether to receive a gene therapy that has not been supported by clinical trial evidence for their SMA type, but independently reviewed and approved by regulators. Second, these regulatory decisions mean that such trials may never be undertaken, which leaves decision makers reliant on arguably lower-quality evidence.

\subsection{DCE Comprehension Questions}

The DCE survey also contained four brief multiple-choice questions aimed at assessing survey participants' understanding of the text describing the treatment attributes. The comprehension questions were presented alongside descriptions of attributes in the introduction to the survey, prior to the DCE choice questions.

\subsection{Experimental Design}

The experimental design of a DCE specifies treatment attribute levels for all attributes for all choice questions. The design can enable independent estimation of each treatment attribute as a predictor of participant treatment preferences and can specify any interactions between attributes that require testing.
It is possible that the level of evidence available for different treatments impacts perceived certainty of treatment benefits in motor and breathing function. The experimental design therefore allowed for testing of the hypothesis that participants will express a stronger preference for a treatment described as having a treatment benefit (defined as stable or better motor function and/or stable or better breathing function) if the treatment has been proven effective in Type 2 and/or 3 SMA than when proven effective in Type 1 SMA only.

An unlabelled D-efficient design with 30 choice questions divided into two blocks of 15 choice sets was generated using NGene software version 1.2.1 [27]. The design included five attributes, each with three attribute levels, and four interactions to examine if evidence in Type 2 and/or 3 SMA (vs Type 1 SMA only) affected strength of preference for treatments with stable or better motor/breathing function.

\subsection{Analysis}

Participant sociodemographic and clinical data were analysed descriptively. Means, standard deviations (SD) and ranges were described for continuous data, and frequencies and proportions were used for categorical data.

The aim of the DCE survey was to evaluate the relative importance of treatment attributes and to establish participants' willingness to trade between attributes. Statistical analysis was conducted using the conditional logit regression model with clustering on respondent level to account for repeated choices in Stata V16.0 [28, 29]. In comparison with a standard conditional logit model, a conditional logit model with clustering yields identical results for beta coefficients but adjusts standard errors to allow for intragroup correlation. The conditional logit model was chosen over more complex choice modelling approaches due to the small study sample size.

A model with main effects for all treatment attributes and pre-specified interaction terms as described above was first tested, showing non-significant interaction effects. Interaction terms were removed from the final model. The reported study results are based on the final model with main effects only. All treatment attributes were treated as categorical variables, meaning that the strength of preference for each attribute level was measured with respect to a reference level. Odds ratios (ORs) and 95\% confidence intervals were calculated from the results of the clustered conditional logit analyses. ORs were used to interpret the importance of each attribute. An alternative-specific constant was added to the model to account for any 'left' bias (i.e. where participants are more likely to choose Treatment A presented on the left over Treatment B on the right).

Responses to comprehension questions were combined into a 'comprehension score', reflecting the number of 
correctly answered questions (maximum score: 4). The association between the comprehension score and DCE choice question completion time was explored using chisquare tests. A sensitivity analysis was conducted to explore whether the direction, the relative order, and the magnitude of estimated coefficients in the conditional logit regression model with clustering were impacted by excluding participants who had incorrectly answered two or more comprehension questions. Results are presented for the total sample and the subgroup of participants who had correctly answered three or more comprehension questions.

\section{Results}

\subsection{Sample Characteristics}

Data collection took place between September 2020 and March 2021. A total of 65 participants completed the survey, of whom 18 were from Portugal, 17 from the Netherlands, 12 from Finland, 11 from Ireland and 7 from Belgium.

Table 2 summarises the demographic and clinical characteristics of adult patients who completed the survey and patients who were children of the caregiver sample. Similar proportions of patients were male $(45 \%)$ and female $(55 \%)$. The overall mean patient age was 31 years old $(\mathrm{SD}=18)$, with a mean age of 42 years $(\mathrm{SD}=12)$ for adult patients self-completing the survey and a mean age of 12 years $(\mathrm{SD}=8)$ for patients whose caregiver completed the survey.

Across the whole sample, most patients had Type 2 SMA (by reported SMA type: $66 \%$; by reported age of symptom onset: $69 \%$ ), and a minority had non-ambulatory Type 3 SMA (31-32\%). The proportion of patients with Type 2 SMA was higher among the patients under the care of the caregiver sample (87\%) than among adult patients self-completing the survey (55-60\%). Reported SMA type varied slightly according to whether participants self- or proxyreported the SMA type or the age of symptom onset.

Most patients were able to sit without assistance (83\%), with few unable to sit (6\%) or able to stand or walk with assistance (11\%). The majority of patients used a wheelchair (92\%). Most patients did not require mechanical breathing support (83\%), some required breathing support for $<16$ h daily (14\%), and very few required breathing support for $>16 \mathrm{~h}$ daily (3\%). Overall, two-fifths of patients were treated with nusinersen $(40 \%)$, with more caregivers reporting patients under their care on nusinersen than adult patients reporting being on nusinersen themselves ( $70 \%$ vs 24\%).

Caregivers in the sample had a mean age of 44 years $(\mathrm{SD}=7)$, with a higher proportion of female caregivers (74\%) than male cargivers (26\%). Most (78\%) caregivers were living with a partner, 9\% were living alone, and 13\% were living with a relative/relatives or had another living arrangement.

\subsection{Treatment Attribute Comprehension Questions}

Most participants (71\%) correctly answered three or more out of four treatment attribute comprehension questions (three correct: 34\%; four correct: 37\%). A minority (29\%) had correctly answered one or two questions (one correct: 6\%; 2 correct: $23 \%$ ). Those who had completed the 15 DCE choice questions in $<160 \mathrm{~s}$ were significantly more likely to have answered comprehension questions incorrectly $\left(\chi^{2}=8.4 ; p<0.05\right)$. Furthermore, none who had completed the choice questions in $<160 \mathrm{~s}$ had correctly answered three or more questions, suggesting a strong association between DCE completion time and engagement with the DCE survey. A sensitivity analysis was therefore conducted to assess the impact of comprehension and completion time on the patient and caregiver treatment preference estimates derived from the study data.

\subsection{Patient and Caregiver Treatment Preferences}

The clustered conditional logit model showed that all treatment attributes were statistically significant, independent predictors of patient and caregiver treatment choices in the total sample (Table 3) and in the subgroup who had correctly answered three or more out of four comprehension questions (Table 4). However, the sensitivity analysis suggested that preferences for some attributes were stronger among the subgroup who had correctly answered most comprehension questions. Further, one attribute level for which no statistically significant preference was found in the total sample, showed a statistically significant difference in the subgroup (once-only intravenous injection with daily tablets vs daily oral liquid). Model results for the total sample are illustrated in Fig. 2.

The model results indicate that motor function had the strongest impact on patient and caregiver choice, followed by breathing function. For the overall study sample, participants were, respectively, 4.8 times and 8.1 times more likely to choose a treatment associated with stable or improved motor function (by one level) compared with a treatment associated with worse motor function, all other things being equal. Similarly, participants were, respectively, 4.3 times and 5.8 times more likely to choose a treatment associated with stable or improved breathing function than one associated with worse breathing function.

Treatment administration, treatment risks and side effects, and demonstrated evidence in SMA types (Types 1, 2 and 3 SMA vs Types 1 and 2 SMA vs Type 1 SMA only) had a smaller influence on choice. Participants were 1.8 times less 
Table 2 Patient characteristics

Patient characteristics

Total, $N=65$

Caregivers (proxy-

reported), $N=23$

Patients (selfreported),

$N=42$

Patient age

Mean (SD)

Min-max

Patient gender

Male, $n(\%)$

Patient living situation, $n(\%)$

Living with partner

Living alone

Living with relative(s)/other

Age at diagnosis (y)

Mean (SD)

Min-max

Age at first symptoms (y)

Mean (SD)

Min-max

Age at first symptoms, $n(\%)$

7-18 mo

SMA categories, $n(\%)$

$>18$ mo to $17 \mathrm{y}$

Reported SMA type, $n(\%)$

Type 2

Type 3

Don't know

Motor function, $n(\%)$

Cannot sit

Sit with some support

Sit independently for few seconds

Sit independently for longer

Stand with assistance

Walk with assistance

Walk independently for few steps

Breathing function (current), $n(\%)$

Mechanical support $>16 \mathrm{~h}$ per day

Mechanical support $<16 \mathrm{~h}$ per day

No mechanical support

SMA treatment, $n(\%)$

Nusinersen

Onasemnogene abeparvovec

Surgery

Other

None

Tools/equipment (past), $n(\%)$

Breathing machine/mechanical ventilation

Feeding tube

Suction machine

Walking frame

Wheelchair

Other

Other conditions, $n(\%)$

$\begin{array}{lll}31.1(18.0) & 11.8(8.4) & 41.7(12.2) \\ 1-62 & 1-44 & 20-62 \\ 29(45) & 10(43) & 19(45)\end{array}$

$14(33)$

$12(29)$

$16(38)$

$4.6(6.0)$

$1.5(0.8)$

$6.4(6.8)$

$0.6-27$

$0.6-4.5$

$0.8-27$

$1.9(2.2)$

$1.1(0.5)$

2.3 (2.6)

$0.5-15.1$

$0.5-2.8$

0.6-15.1

$45(69)$

20 (87)

$25(60)$

$20(31)$

3 (13)

$17(40)$

43 (66)

20 (87)

23 (55)

21 (32)

3 (13)

18 (43)

1 (2)

0 (0)

1 (2)

4 (6)

20 (31)

2 (9)

2 (5)

12 (18)

22 (34)

2 (3)

3 (5)

2 (3)

7 (30)

13 (31)

8 (19)

14 (33)

2 (5)

$1(2)$

$2(5)$

2 (3)

2 (5)

9 (14)

54 (83)

7 (17)

$33(79)$

$26(40)$

$10(24)$

0 (0)

28 (43)

6 (9)

18 (28)

$0(0)$

2 (9)

21 (91)

0 (0)

0 (0)

$19(45)$

9 (39)

5 (12)

$13(31)$

$16(25) \quad 6(26) \quad 10(24)$

$3(5)$

0 (0)

3 (7)

4 (6)

$0(0)$

$4(10)$

1 (2)

60 (92)

1 (4)

0 (0)

19 (29)

$21(91)$

39 (93)

6 (26)

13 (31)

15 (23)

2 (9)

13 (31)

$S D$ standard deviation, SMA spinal muscular atrophy 
Table 3 Results of a clustered conditional logit model of patient and caregiver preferences: total sample

\begin{tabular}{|c|c|c|c|c|c|c|c|c|c|}
\hline Attributes and levels & \multirow[t]{2}{*}{ Coeff. } & \multirow[t]{2}{*}{ Robust std error } & \multirow[b]{2}{*}{ nn) } & \multirow[t]{2}{*}{$p$ value } & \multicolumn{2}{|c|}{$\begin{array}{l}95 \% \text { CI } \\
\text { (coefficients) }\end{array}$} & \multirow[t]{2}{*}{ Odds ratios } & \multicolumn{2}{|c|}{$\begin{array}{l}95 \% \text { CI } \\
\text { (odds ratios) }\end{array}$} \\
\hline \multicolumn{5}{|c|}{ Alternative-specific constant-reference category: Treatment A (left column) } & & & & & \\
\hline Treatment A (left column) & 0.172 & 0.093 & 1.840 & 0.065 & -0.011 & 0.355 & 1.188 & 0.989 & 1.427 \\
\hline \multicolumn{10}{|c|}{ Motor function-reference category: worse by one level } \\
\hline Stable & 1.577 & 0.158 & 9.950 & 0.000 & 1.267 & 1.888 & 4.842 & 3.549 & 6.606 \\
\hline Improved by one level & 2.095 & 0.217 & 9.650 & 0.000 & 1.670 & 2.521 & 8.127 & 5.310 & 12.440 \\
\hline \multicolumn{10}{|l|}{ Breathing function-reference category: worse } \\
\hline Stable & 1.458 & 0.181 & 8.050 & 0.000 & 1.103 & 1.813 & 4.297 & 3.013 & 6.129 \\
\hline Better & 1.756 & 0.239 & 7.350 & 0.000 & 1.287 & 2.224 & $\mathbf{5 . 7 8 7}$ & 3.624 & 9.244 \\
\hline \multicolumn{10}{|c|}{ Treatment administration—reference category: oral liquid taken once daily at home } \\
\hline Injection in spine in hospital every 4 months & -0.587 & 0.153 & -3.830 & 0.000 & -0.887 & -0.287 & 0.556 & 0.412 & 0.751 \\
\hline $\begin{array}{l}\text { One-off injection in hospital with daily oral tablets } \\
\text { for } 2 \text { months }\end{array}$ & -0.131 & 0.113 & -1.160 & 0.244 & -0.352 & 0.090 & 0.877 & 0.703 & 1.094 \\
\hline \multicolumn{10}{|c|}{ Risk and side effects—reference category: $17 \%$ risk of diarrhoea and rash } \\
\hline $\begin{array}{l}\text { Fever, headache, vomiting, body pain every } \\
4 \text { months }\end{array}$ & 0.033 & 0.097 & 0.340 & 0.737 & -0.158 & 0.223 & 1.033 & 0.854 & 1.249 \\
\hline $\begin{array}{l}10 \% \text { risk of liver injury. Fatigue, headache, nausea } \\
\text { for } 2 \text { months }\end{array}$ & -0.455 & 0.130 & -3.490 & 0.000 & -0.710 & -0.199 & 0.635 & 0.492 & 0.819 \\
\hline \multicolumn{10}{|c|}{ Treatment evidence-reference category: demonstrated in Types 1, 2 and 3 SMA } \\
\hline Demonstrated in Types 1 and 2 SMA & -0.161 & 0.123 & -1.310 & 0.190 & -0.402 & 0.080 & 0.851 & 0.669 & 1.083 \\
\hline Demonstrated in Type 1 SMA & -0.843 & 0.185 & -4.550 & 0.000 & -1.206 & -0.479 & 0.431 & 0.299 & 0.619 \\
\hline
\end{tabular}

Model statistics: Wald $\chi^{2}=256.51 ; p<0.001$

Bold indicates statistically significant at 0.05 level

CI confidence interval, SMA spinal muscular atrophy

likely to choose a treatment administered via an intrathecal injection in hospital every 4 months than a treatment taken orally once daily at home, all else being equal. Participants were equally likely to prefer the oral therapy or the onetime intravenous treatment. Participants preferred to avoid adverse events as would be expected. They were 1.6 times less likely to choose a treatment associated with a risk of liver injury, and fatigue, headache and/or nausea compared with a treatment associated with a risk of diarrhoea and rash. Participants considered treatments associated with headache, vomiting and/or body pain every 4 months as equally bad as a treatment with a risk of diarrhoea and rash.

Participants were 2.3 times less likely to choose a treatment for which effectiveness had been demonstrated in Type 1 SMA only compared with a treatment with demonstrated effectiveness in Types 1, 2 and 3 SMA. Participants were no more or less likely to choose a treatment with demonstrated effectiveness in Types 1 and 2 SMA compared with a treatment with demonstrated effectiveness in Types 1, 2 and 3 SMA.

For the subgroup of participants who correctly answered at least three comprehension questions, the results are broadly the same. The relative ordering of importance of the attributes was the same for this subgroup. The ORs for most of the attributes were further from 1 . In this sensitivity analysis, participants were 1.4 times less likely to choose the one-off injection with daily tablets for 2 months than a daily oral treatment, which is a difference that did not emerge for the group overall.

\section{Discussion}

Three treatments for SMA have recently been approved in Europe. Understanding patient and caregiver preferences for different treatment characteristics has therefore become increasingly relevant to payers and clinical decision makers. This stated preference study was designed to examine adult patient and caregiver preferences for treatments in Type 2 and non-ambulatory Type 3 SMA in five European countries. The results show that patient and caregiver treatment choices were most strongly influenced by treatment effectiveness in terms of stable and improved motor function. The extent to which participants were willing to make trade-offs increased in stepwise fashion from stable to improved motor function, compared with worse motor function. Treatment effectiveness in relation to breathing function was the second most important attribute to patients and caregivers. Participants valued stable breathing function and stable motor function similarly. However, they appeared to value better 
Table 4 Results of a clustered conditional logit model of patient and caregiver preferences: subgroup who correctly answered at least three out of four comprehension questions

\begin{tabular}{|c|c|c|c|c|c|c|c|c|c|}
\hline Attributes and levels & \multirow[t]{2}{*}{ Coeff. } & \multirow[t]{2}{*}{ Robust std error } & \multirow[b]{2}{*}{ n) } & \multirow[t]{2}{*}{$p$ value } & \multicolumn{2}{|c|}{$\begin{array}{l}95 \% \text { CI } \\
\text { (coefficients) }\end{array}$} & \multirow[t]{2}{*}{ Odds ratios } & \multicolumn{2}{|c|}{$\begin{array}{l}95 \% \text { CI } \\
\text { (odds ratios) }\end{array}$} \\
\hline \multicolumn{5}{|c|}{ Alternative-specific constant-reference category: Treatment A (left column) } & & & & & \\
\hline Treatment A (left column) & 0.018 & 0.109 & 0.170 & 0.868 & -0.196 & 0.233 & 1.018 & 0.822 & 1.262 \\
\hline \multicolumn{10}{|c|}{ Motor function-reference category: worse by one level } \\
\hline Stable & 1.837 & 0.220 & 8.360 & 0.000 & 1.406 & 2.267 & 6.277 & 4.081 & 9.655 \\
\hline Improved by one level & 2.458 & 0.281 & 8.750 & 0.000 & 1.907 & 3.009 & 11.685 & 6.736 & 20.271 \\
\hline \multicolumn{10}{|l|}{ Breathing function-reference category: worse } \\
\hline Stable & 1.790 & 0.228 & 7.870 & 0.000 & 1.344 & 2.236 & 5.987 & 3.833 & 9.352 \\
\hline Better & 2.139 & 0.308 & 6.950 & 0.000 & 1.535 & 2.742 & 8.489 & 4.643 & 15.523 \\
\hline \multicolumn{10}{|c|}{ Treatment administration—reference category: oral liquid taken once daily at home } \\
\hline Injection in spine in hospital every 4 months & -0.814 & 0.157 & -5.200 & 0.000 & -1.121 & -0.507 & 0.443 & 0.326 & 0.602 \\
\hline $\begin{array}{l}\text { One-off injection in hospital with daily oral tablets } \\
\text { for } 2 \text { months }\end{array}$ & -0.339 & 0.134 & -2.520 & 0.012 & -0.602 & -0.076 & 0.713 & 0.548 & 0.927 \\
\hline \multicolumn{10}{|c|}{ Risk and side effects-reference category: $17 \%$ risk of diarrhoea and rash } \\
\hline $\begin{array}{l}\text { Fever, headache, vomiting, body pain every } \\
4 \text { months }\end{array}$ & 0.089 & 0.131 & 0.680 & 0.498 & -0.168 & 0.346 & 1.093 & 0.845 & 1.413 \\
\hline $\begin{array}{l}10 \% \text { risk of liver injury. Fatigue, headache, nausea } \\
\text { for } 2 \text { months }\end{array}$ & -0.519 & 0.189 & -2.740 & 0.006 & -0.890 & -0.148 & 0.595 & 0.411 & 0.863 \\
\hline \multicolumn{10}{|c|}{ Treatment evidence-reference category: demonstrated in Types 1,2 \& 3 SMA } \\
\hline Demonstrated in Types 1 and 2 SMA & -0.268 & 0.148 & -1.820 & 0.069 & -0.558 & 0.021 & 0.765 & 0.573 & 1.021 \\
\hline Demonstrated in Type 1 SMA & -0.783 & 0.222 & -3.520 & 0.000 & -1.218 & -0.347 & 0.457 & 0.296 & 0.707 \\
\hline
\end{tabular}

Model statistics: Wald $\chi^{2}=273.96 ; p<0.001$

Bold indicates statistically significant at 0.05 level

CI confidence interval, SMA spinal muscular atrophy

breathing function less than better motor function. These findings are consistent with the clinical characteristics of the patient sample in this study. Most patients were able to sit but could not stand or walk and did not require mechanical breathing support, so it is evident why they would place a high value on maintaining or improving their motor function and maintaining breathing function, but not necessarily improving breathing function.

All SMA treatments aim to stabilise or improve motor and breathing function. However, the treatment administration, treatment risks and side effects and treatment evidence attributes in this study were based on differences in the treatment profiles for nusinersen, risdiplam and onasemnogene abeparvovec. The intrathecal injections for nusinersen can cause flu-like symptoms such as fever, headache, vomiting and bodily pain in patients [11]. Approximately one in six patients who received risdiplam in clinical trials experienced diarrhoea and/or rash [26]. Onasemnogene abeparvovec has been shown to cause liver injury in $10 \%$ of patients, along with fatigue, headache and nausea due to steroids taken alongside the gene therapy [13]. The study results showed that participants also considered these differentiating treatment characteristics when making their treatment choices. Participants preferred oral daily therapy over receiving the treatment via an intrathecal injection. No statistically significant difference was found between preferences for a onetime intravenous infusion combined with oral tablets for 2 months and daily oral therapy (in the total sample analysis). Regarding treatment side effects, participants preferred to avoid a $10 \%$ risk of liver injury compared with a $17 \%$ risk of diarrhoea and rash. However, participants were no more or less likely to choose a treatment with side effects associated with intrathecal injections (fever, headache, vomiting and/ or body pain) compared with a treatment with a $17 \%$ risk of diarrhoea and rash. Lastly, participants preferred to avoid treatments where evidence of treatment efficacy for their type of SMA has not been demonstrated.

The sensitivity analysis with the subgroup of participants who had correctly answered most comprehension questions showed that preferences for some attributes, in particular motor and breathing function, were stronger among this subgroup. This subgroup also had a slight preference for avoiding a one-time intravenous injection accompanied by oral tablets for 2 months compared with daily oral therapy, whereas this difference was not statistically significant in the total sample. These findings suggest that estimates derived from the total sample were attenuated due to measurement 


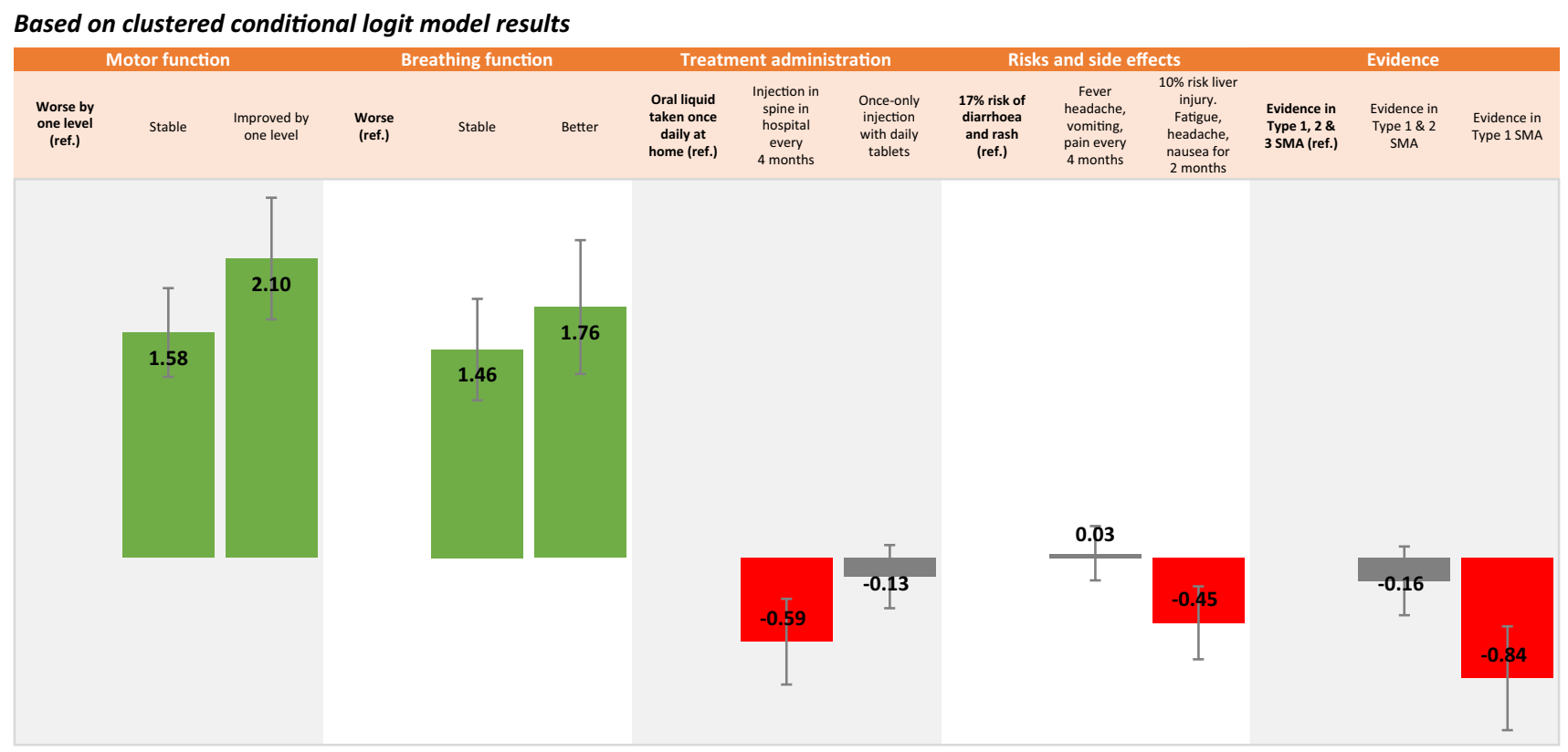

Error bars indicate $95 \%$ confidence intervals

Fig. 2 Estimated patient and caregiver preference weights (based on model beta coefficients) for treatment attributes. Green and red bars represent significant positive and negative preferences, grey bars are not significant (based on clustered conditional logit model results)

error caused by a minority of participants who were less engaged with the survey.

The DCE method forces participants to make choices between hypothetical treatments. Their choices help us to understand what is most important, relevant but less important, and what is not (or less) relevant to people's treatment choices. The study results showed that treatment effectiveness, as indicated by stabilised or improved motor and breathing function, was most important to patients and caregivers. In addition, avoiding intrathecal injections, treatments associated with risk of liver injury, and treatments with no demonstrated efficacy in people with their SMA type were also relevant, albeit less important. There are similarities between the results of this study and previous DCEs with patients with SMA and caregivers of patients with SMA in the UK and US [19, 20]. For example, previous research also found that patients place value on the stabilisation and improvement of motor and respiratory function, despite variations in study design [19, 20].

We believe it is important to communicate patient and caregiver preferences to decision makers, healthcare providers and other stakeholders involved in the development of new treatments. This study reports data from the Netherlands, Belgium, Finland, Ireland, and Portugal, combined together into a single analysis. The data could support national decision making in the respective countries. In a rare disease like SMA, it is often not feasible to capture samples large enough for a separate analysis in each country.

\subsection{Study Limitations}

This study has several limitations that should be considered when interpreting the results. First, the sample consisted of a mix of adult patients and caregivers of patients with Type 2 and non-ambulatory Type 3 SMA. Due to the small sample size, differences in treatment preferences between patients with different clinical characteristics (e.g. reported level of patient motor or breathing function) could not be examined. Similarly, differences in preferences between adult patients and caregivers were also not examined. Findings that are not statistically significant may also be less robust. No power calculations were undertaken to determine the sample size, partly because this is a rare disease and so the sample size was determined by availability of participants. The interaction effects between treatment evidence and treatment effectiveness were not statistically significant in both the total sample and subgroup analysis of participants who correctly answered most comprehension questions. It is possible that a study with a larger sample size and more statistical power to detect any potential effects would have found statistically significant interactions or more significant main effects. Secondly, the dropout rate during survey completion was high, with $32 \%$ of those who had consented to take part in the survey dropping out before survey completion. To examine dropout patterns, patient characteristics of those who had dropped out and those who completed the survey were compared with those who had completed questions on patient characteristics at the beginning of the 
main survey ( $85 \%$ of those who had consented). There were no statistically significant differences in patient characteristics between those who completed the survey and those who dropped out. Thirdly, this study did not seek patient feedback on the survey design, which was adapted from a previous study, nor was a pilot study conducted to assess patient comprehension of the survey. Seeking patient input may have allowed us to design the survey in such a way that it was more specific to their needs and concerns. The survey included an attribute that described whether a treatment was supported by clinical trial evidence in Type 1,2 and/or 3 SMA versus Type 1 SMA only. This was included to understand whether participants were concerned about this uncertainty in the evidence base for the type of SMA that they or the person they care for have. However, our ability to interpret the importance of this attribute is limited by the fact that the choices provide average outcomes for motor and respiratory function. Participants may have been left wondering whether the lack of evidence for their SMA type would have impacted the treatment benefit to them. The non-significant interaction between treatment evidence and treatment effectiveness attributes might indicate that the evidence attribute did not impact the relative perceived value of the effectiveness attributes. This might suggest that participants did not interpret lack of evidence in their SMA type as impacting treatment benefit to them or the patient they care for, although as noted earlier, the study may have been underpowered to test for interaction effects. Therefore, the study may have underestimated how important the treatment evidence attribute is to study participants because it was combined with fixed average treatment outcomes in the choice sets. Validation through patient feedback would have enhanced the robustness of the study. The survey recruited patients and caregivers anonymously via patient advocacy associations. Participant identity, and patient and caregiver status were not verified, and recruiting via associations only may have biased the sample to only include those who are members. It is possible that members of patient advocacy groups would respond differently to these choice questions than non-members, perhaps because they may be more proactive in seeking help or managing their condition. People who choose not to join advocacy groups may have a different perspective on the care they receive and the value of different outcomes. Lastly, the survey attributes were limited to features of currently available treatments, but in principle could have included features of treatments in development (or even combinations of current SMA treatments). All three treatments represent highly innovative therapies that are addressing unmet need. Stabilising or improving function, differing treatment options in terms of mode of administration, and the potential for patients to live with SMA for many years are all important innovations.

\section{Conclusion}

Adult patients and caregivers of patients with Type 2 and non-ambulatory Type 3 SMA place substantial value on accessing effective therapies to relieve the burden of their condition or the condition of the patient they care for. The present study findings demonstrate the importance of motor and breathing function to patients and caregivers of this SMA population. Not only did participants value improvements in motor and breathing function, they also placed considerable value on maintaining these functions. Whilst less important than treatment effectiveness, patients and caregivers also made choices to avoid intrathecal injections, treatments described as involving risk of liver injury in combination with fatigue, headache and nausea, and treatments with demonstrated effectiveness in Type 1 SMA only.

Acknowledgements We would like to thank study participants for their help by taking part in the survey, SMA Belgium, SMA Ireland, SMA Finland, Associação Portuguesa de Neuromusculares and Vereniging Spierziekten Nederland for their valuable contribution to the study, and Roche colleagues Maitiu O'Nuallain, Merja Hautala, Lauri Siljander, Luuk den Boer, Joost Geenen and Benedicte Mast for their support and contribution.

\section{Declarations}

Disclosure statement This article is published in a special edition journal supplement wholly funded by F. Hoffmann-La Roche Ltd.

Funding This study was sponsored by F. Hoffmann-La Roche Ltd.

Conflicts of interest/competing interests SHL, CL and AJL are employees of Acaster Lloyd Consulting Ltd, who were commissioned by F. Hoffmann-La Roche Ltd to conduct this study. During the preparation of this manuscript, YM and AC were employed by F. HoffmannLa Roche Ltd.

Ethics approval This study was reviewed and received exempt status determination by the Western Institutional Review Board (WIRB) on 19 August 2020 (submission number: 2639333-44664387). All participants gave informed consent prior to taking part in the study.

Consent to participate Freely given, informed consent to participate in the study was obtained from participants prior to participation in the study.

Consent for publication Freely given, informed consent to the scientific publication of the results of this research was obtained from participants prior to participation in the study.

Availability of data and material The sponsor is adhering to current EU requirements, so will not make individual deidentified participant data available; however, the study protocol will be made available upon reasonable request to the corresponding author.

Code availability Not available. 
Author contributions SHL, CL and AJL designed the study. SHL analysed the data. SHL, CL and AJL interpreted the data and drafted the manuscript. YM and AC contributed to the design of the study, interpreted the data and revised the draft critically for important intellectual content. All authors approved the final version of the manuscript and agree to be accountable for all aspects of the work in ensuring that questions related to the accuracy or integrity of any part of the work are appropriately investigated and resolved.

Open Access This article is licensed under a Creative Commons Attribution-NonCommercial 4.0 International License, which permits any non-commercial use, sharing, adaptation, distribution and reproduction in any medium or format, as long as you give appropriate credit to the original author(s) and the source, provide a link to the Creative Commons licence, and indicate if changes were made. The images or other third party material in this article are included in the article's Creative Commons licence, unless indicated otherwise in a credit line to the material. If material is not included in the article's Creative Commons licence and your intended use is not permitted by statutory regulation or exceeds the permitted use, you will need to obtain permission directly from the copyright holder. To view a copy of this licence, visit http://creativecommons.org/licenses/by-nc/4.0/.

\section{References}

1. D’Amico A, Mercuri E, Tiziano FD, Bertini E. Spinal muscular atrophy. Orphanet J Rare Dis. 2011;6:71. https://doi.org/10.1186/ 1750-1172-6-71.

2. Butchbach MER. Copy number variations in the survival motor neuron genes: Implications for spinal muscular atrophy and other neurodegenerative diseases. Front Mol Biosci. 2016;3:7. https:// doi.org/10.3389/fmolb.2016.00007.

3. Mercuri E, Finkel R, Montes J, Mazzone ES, Sormani MP, Main M, et al. Patterns of disease progression in type 2 and 3 SMA: implications for clinical trials. Neuromuscul Disord. 2016;26(2):126-31. https://doi.org/10.1016/j.nmd.2015.10.006.

4. Finkel RS, McDermott MP, Kaufmann P, Darras BT, Chung WK, Sproule DM, et al. Observational study of spinal muscular atrophy type I and implications for clinical trials. Neurology. 2014;83(9):810-7. https://doi.org/10.1212/WNL.0000000000 000741.

5. Annoussamy M, Seferian AM, Daron A, Péréon Y, Cances C, Vuillerot C, et al. Natural history of Type 2 and 3 spinal muscular atrophy: 2-year NatHis-SMA study. Ann Clin Transl Neurol. 2021;8(2):359-73. https://doi.org/10.1002/acn3.51281.

6. Chabanon A, Seferian AM, Daron A, Péréon Y, Cances C, Vuillerot $\mathrm{C}$, et al. Prospective and longitudinal natural history study of patients with Type 2 and 3 spinal muscular atrophy: baseline data NatHis-SMA study. PLoS ONE. 2018. https://doi.org/10.1371/ journal.pone.0201004.

7. Qian Y, McGraw S, Henne J, Jarecki J, Hobby K, Yeh WS. Understanding the experiences and needs of individuals with Spinal Muscular Atrophy and their parents: a qualitative study. BMC Neurol. 2015;15:217. https://doi.org/10.1186/s12883-015-0473-3.

8. Rouault F, Christie-Brown V, Broekgaarden R, Gusset N, Henderson D, Marczuk P, et al. Disease impact on general well-being and therapeutic expectations of European Type II and Type III spinal muscular atrophy patients. Neuromuscul Disord. 2017;27(5):42838. https://doi.org/10.1016/j.nmd.2017.01.018.

9. McGraw S, Qian Y, Henne J, Jarecki J, Hobby K, Yeh WS. A qualitative study of perceptions of meaningful change in spinal muscular atrophy. BMC Neurol. 2017;17(1):68. https://doi.org/ 10.1186/s12883-017-0853-y.
10. Gusset N, Stalens C, Stumpe E, Klouvi L, Mejat A, Ouillade MC, et al. Understanding European patient expectations towards current therapeutic development in spinal muscular atrophy. Neuromuscul Disord. 2021;31(5):419-30. https://doi.org/10.1016/j. nmd.2021.01.012.

11. European Medicines Agency. SPINRAZA Summary of product characteristics. 2021. European Medicines Agency. https://www. ema.europa.eu/en/documents/product-information/spinraza-eparproduct-information_en.pdf. Accessed 16 Jun 2020

12. Zanetta C, Riboldi G, Nizzardo M, Simone C, Faravelli I, Bresolin $\mathrm{N}$, et al. Molecular, genetic and stem cell-mediated therapeutic strategies for spinal muscular atrophy (SMA). J Cell Mol Med. 2014;18(2):187-96. https://doi.org/10.1111/jcmm.12224.

13. European Medicines Agency. ZOLGENSMA Summary of product characteristics. European Medicines Agency. 2021. https://www. ema.europa.eu/en/documents/product-information/zolgensmaepar-product-information_en.pdf. Accessed 27 Apr 2021.

14. Mendell JR, Al-Zaidy S, Shell R, Arnold WD, Rodino-Klapac LR, Prior TW, et al. Single-dose gene-replacement therapy for spinal muscular atrophy. N Engl J Med. 2017;377:1713-22. https://doi. org/10.1056/NEJMoa1706198.

15. Strauss K, Muntoni F, Farrar M, Saito K, Mendell J, Servais L, et al. Onasemnogene Abeparvovec Gene Therapy in Presymptomatic Spinal Muscular Atrophy (SMA): SPR1NT Study Update in Children with 2 Copies of SMN2 (4190). Neurology. 2021;96.

16. Strauss K, Muntoni F, Farrar M, Saito K, Mendell J, Servais L, et al. Onasemnogene Abeparvovec Gene Therapy in Presymptomatic Spinal Muscular Atrophy (SMA): SPR1NT Study Update in Children with 3 Copies of SMN2 (4163). Neurology. 2021;96.

17. Darras BT, Masson R, Mazurkiewicz-Bełdzińska M, Rose K, Xiong H, Zanoteli E, et al. FIREFISH Part 2: 24-month Efficacy and Safety of Risdiplam in Infants with Type 1 Spinal Muscular Atrophy (SMA) (4126). Neurology. 2021;96.

18. Mercuri E, Barisic N, Boespflug-Tanguy O, Day JW, Deconinck N, Kostera-Pruszczyk A, et al. SUNFISH Part 2: Efficacy and Safety Of Risdiplam (RG7916) in Patients with Type 2 or Nonambulant Type 3 Spinal Muscular Atrophy (SMA) (1260). Neurology. 2020;96.

19. Lo SH, Paracha N, Gorni K, Lloyd A. PRO93 do caregivers and patients value the avoidance of lumbar punctures in spinal muscular atrophy? A stated preference survey. Value Heal. 2020. https:// doi.org/10.1016/j.jval.2020.04.1314.

20. Monnette A, Chen E, Hong D, Bazzano A, Dixon S, Arnold WD, et al. Treatment preference among patients with spinal muscular atrophy (SMA): a discrete choice experiment. Orphanet J Rare Dis. 2021;16:36. https://doi.org/10.1186/s13023-020-01667-3.

21. Hauber AB, González JM, Groothuis-Oudshoorn CGM, Prior T, Marshall DA, Cunningham C, et al. Statistical methods for the analysis of discrete choice experiments: a report of the ISPOR conjoint analysis good research practices task force. Value Health. 2016;19(4):300-15. https://doi.org/10.1016/j.jval.2016.04.004.

22. Johnson FR, Lancsar E, Marshall D, Kilambi V, Mühlbacher A, Regier DA, et al. Constructing experimental designs for discretechoice experiments: report of the ISPOR conjoint analysis experimental design good research practices task force. Value Health. 2013;16:3-13. https://doi.org/10.1016/j.jval.2012.08.2223.

23. De Bekker-Grob EW, Ryan M, Gerard K. Discrete choice experiments in health economics: a review of the literature. Health Econ. 2012;21(2):145-72. https://doi.org/10.1002/hec.1697.

24. U.S. Food and Drug Administration. Approval Package for Spinraza Injection, $2.4 \mathrm{mg} / \mathrm{mL}$. 2016. https://www.accessdata.fda. gov/drugsatfda_docs/nda/2016/209531Orig1s000Approv.pdf. Accessed 27 Apr 2021.

25. Mercuri E, Darras BT, Chiriboga CA, Day JW, Campbell C, Connolly AM, et al. Nusinersen versus sham control in later-onset 
spinal muscular atrophy. N Engl J Med. 2018;378(7):625-35. https://doi.org/10.1056/NEJMoa1710504.

26. European Medicines Agency. Evrysdi summary of product characteristics. 2021. European Medicines Agency. https://www.ema. europa.eu/en/documents/product-information/evrysdi-epar-produ ct-information_en.pdf. Accessed 07 Oct 2021.

27. ChoiceMetrics. Ngene 1.2 USER MANUAL \& REFERENCE GUIDE The Cutting Edge in Experimental Design. 2018. http:// www.choice-metrics.com/NgeneManual120.pdf. Accessed 27 Apr 2021.
28. McFadden D (1973) Conditional logit analysis of qualitative choice behavior. In: Zarembka P (ed) Frontiers in econometrics. Academic Press, pp. 105-142.

29. StataCorp. Stata Statistical Software: Release 16. College Station, TX: StataCorp LLC.; 2019. https://www.stata.com/support/faqs/ resources/citing-software-documentation-faqs/. Accessed $27 \mathrm{Apr}$ 2021. 